\title{
Feedback Slew Algorithms for Prolate Spinners Using Single-Thruster
}

\author{
Juntian $\mathrm{Si}^{1}$, Yang Gao, Abadi Chanik \\ Surrey Space Centre, University of Surrey, Guildford GU2 7XH, United Kingdom
}

\begin{abstract}
A number of low-cost open-loop slew control algorithms have been developed for prolate spinning spacecraft using single-thruster actuation. Robustness analysis indicates that these algorithms have high sensitiveness over thruster firing time error, spacecraft inertia error, and especially spin rate perturbations. This paper proposed two novel feedback slew algorithms, Feedback Half-Cone and Feedback Sector-Arc Slew, built on the existing open-loop algorithms and they use attitude and angular velocity feedback to improve robustness. As presented, after the first thruster actuation initiate the spinaxis precession, the feedback slew algorithms take attitude and spin-rate feedback to estimate the angular momentum and predict the spin-axis attitude during the slew. These techniques contribute to improve the cancelation thrust impulse accuracy and reduce the final nutation error. Simulations for a Penetrator mission scenario validate these feedback algorithms and show their slew performance and robustness over the perturbations mentioned above. It is proved that the attitude feedback greatly improves the slew accuracy and robustness.
\end{abstract}

Keywords: prolate penetrator, attitude control, feedback control, attitude dynamics, spinning spacecraft, single thruster

\section{Introduction}

The attitude control algorithms proposed in this paper are discussed for Penetrator mission concept: a cylindrical missile-shaped projectile spinning around its minor axis of inertia performs a $90^{\circ}$ spin axis reorientation manoeuvre to impact with a celestial body, burying itself into the subsurface for investigation. Japanese mission Lunar-A [1] and British MoonLITE mission [2] using above Penetrator concept both aimed to study the lunar subsurface. Spin stabilization is usually taken as it is a relatively low-cost means of stabilization. With only one thruster mounted perpendicular to the spin-axis, the attitude slew can be achieved using state-of-the-art single-thruster slew algorithms. Existing research [3-6] on the prolate spinning spacecraft attitude manoeuvre has developed a series of slew algorithms using single-thruster in two categories: Half-cone derived algorithms and Pulse-train algorithms. Halfcone derived algorithms consist of Half-Cone (HC), Multi Half-Cone (MHC), Dual Half-Cone (DHC), Extended Half-Cone (EHC), Sector Arc Slew (SAS), Multi Sector Arc (MSA) slew, using the precession behaviour of a spinning prolate spacecraft. Pulse-train algorithms consist of Rhumb Line (RL) and Spin-Synch (SS) algorithms, which use a train of uniform torque pulses to achieve the attitude manoeuvre. The timing between two torque pulses is roughly (for Rhumb Line) or exactly (for SpinSynch) equal to the spin period. Pulse-train algorithms can also be used for oblate spacecraft. Based on the Half-Cone slew using single-thruster, similar Half-Cone slew method using single-magnetorquer [7] are also developed aimed to apply on STRaND-1 [8] mission.

Previous analysis [3] concludes that within the range of manoeuvre angles the mission required, SAS, MSA, DC and EHC slews can achieve accurate slews. However above Half-Cone derived algorithms are all open-loop control without attitude feedback and the robustness analysis [9] indicates that they all show high sensitiveness over thruster firing time error, spacecraft inertia error, and especially spin

\footnotetext{
${ }^{1}$ Corresponding author

Telephone: +44 7581211376, Fax: +4401483689503
} 
rate perturbations. With about $1 \%$ of the spin-rate perturbation, the slew error would exceed over $50^{\circ}$, which can be treated as the failure of the manoeuvre. In order to improve the robustness of singlethruster slews, research is motivated to use attitude and angular velocity feedbacks to develop novel feedback slew algorithms. Feedback Half-Cone Slew and Feedback Sector-Arc Slew are proposed in this paper based on the open-loop HC and SAS slews. For these two novel algorithms, angular momentum estimation and latter spin-axis attitude prediction are the key techniques and the adjusted timing of precession cancellation impulse in the last revolution of the manoeuvre contributes to a better slew accuracy.

Based on the numerical simulation for a given Penetrator mission scenario, thorough comparative analysis of the novel feedback algorithms and their corresponding open-loop algorithms is given focusing on spin-axis slew accuracy, slew duration and energy consumption.

The rest of this paper is organized as follows. Section 2. defines the coordinate frames of this research and Section 3. briefly presents the existing open-loop HC and SAS slew algorithms. Section 4. introduces the novel feedback HC and Feedback SAS slew algorithms as well as the techniques of angular momentum estimation and latter spin-axis attitude prediction using attitude feedback. Section 5. presents the slew performance and robustness analysis of the feedback algorithms compared with their corresponding open-loop ones, and the thorough comparison between these two feedback slews, which indicates a possible methodology to choose slew algorithms for a certain space mission for space engineers. In the end, Section 6. summarizes the conclusions of the paper.

\section{Mathematical Background}

The fundamental mathematical background of the spinners' spin axis attitude manoeuvre is the rigid body rotational Kinematics and Dynamics. For a rigid body, the rotational movement driven by a torque applied on it is defined as Euler's Moment Equation, explained and demonstrated in Wertz [10]'s equation:

$$
\boldsymbol{T}=\dot{\boldsymbol{H}}_{R I}=\dot{\boldsymbol{H}}_{S F B}+\boldsymbol{\omega} \times \boldsymbol{H}
$$

For a prolate axisymmetric spinner, the angular velocity vector $\boldsymbol{\omega}$, spin axis $\mathrm{Z}$ and angular momentum vector $\boldsymbol{H}$ are in the same plane and this plane rotates about $\boldsymbol{H}$ with angular velocity $\omega_{H}$. In external torque free case, the magnitudes and the relative positions of these vectors are constant during the rotation. $\lambda$ is the inertia ratio which equals to $I_{Z} / I_{t}$. For a prolate spinner, $0<\lambda<1$, and the relationships between these vectors are illustrated in Fig. 1. In Fig.1, $\boldsymbol{\omega}$ can be geometrically decomposed in two sets of components:

(1) $\boldsymbol{\omega}_{Z}$ and $\boldsymbol{\omega}_{X Y}$, which are perpendicular decompositions in SFB frame $\mathrm{Z}$ axis and XOY plane.

(2) $\boldsymbol{\omega}_{N}$ and $\boldsymbol{\omega}_{H}$, which are the non-perpendicular decompositions in $\mathrm{Z}$ axis and $\boldsymbol{H}$ vector direction, with an enclosed angle $\theta$ defined as the nutation angle.

This plane as well as $\mathrm{Z}$ axis and $\boldsymbol{\omega}$ rotates around the angular momentum $\boldsymbol{H}$ with angular velocity $\boldsymbol{\omega}_{H}$, namely inertial nutation rate. The body nutation rate $\boldsymbol{\omega}_{N}$ is the rotation rate of any point fixed in the spacecraft around $Z$ axis relative to the orientation of $\boldsymbol{H}$. In inertial space, $\boldsymbol{\omega}$ rotates around $\boldsymbol{H}$ on a cone called the space cone. Similarly, $\omega$ rotates around $Z$ on a cone called the body cone. In total, the motion of the spacecraft is visualized as the body cone rolling on the space cone without slipping. 


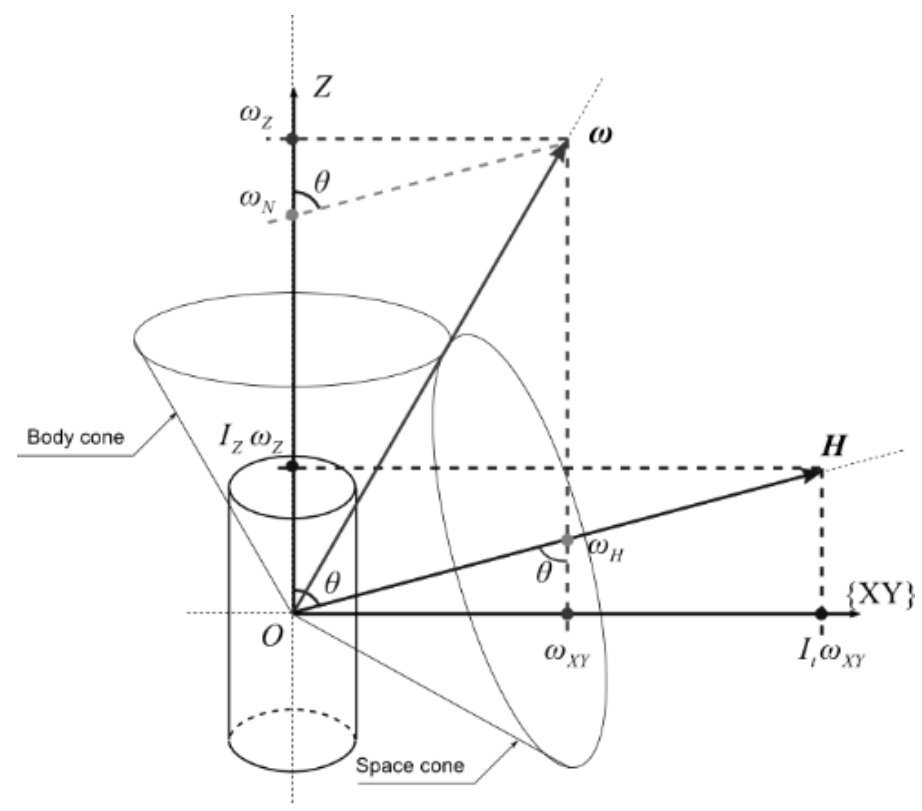

Fig. 1. precession in Z-H plane illustration

The relationship between $\omega_{H}, \omega_{N}$ and $\omega_{Z}$ can be derived as follows:

$$
\begin{gathered}
\omega_{H}=\frac{\|\boldsymbol{H}\| \omega_{X Y}}{I_{t} \omega_{X Y}}=\frac{\|\boldsymbol{H}\|}{I_{t}}=\frac{I_{Z} \omega_{Z}}{\cos (\theta) I_{t}}=\frac{\lambda \omega_{Z}}{\cos (\theta)} \\
\tan (\theta)=\frac{\omega_{X Y}}{\omega_{Z}-\omega_{N}}=\frac{I_{t} \omega_{X Y}}{I_{Z} \omega_{Z}} \Leftrightarrow \\
\omega_{Z}-\omega_{N}=\omega_{X Y} \frac{I_{Z} \omega_{Z}}{I_{t} \omega_{X Y}}=\lambda \omega_{Z} \Leftrightarrow \\
\omega_{N}=(1-\lambda) \omega_{Z}
\end{gathered}
$$

where $I_{t}$ and $I_{Z}$ are the inertia around $\mathrm{X} / \mathrm{Y}$ axis and around $\mathrm{Z}$ axis respectively.

\section{State-of-the-art Open-loop Half-Cone and Sector-Arc Slew Algorithms}

\subsection{Half-Cone Slew}

For Half-Cone Slew, it is assumed that the prolate spacecraft is initially in a pure spin around SFB Zaxis. The thruster position and thrust direction are chosen to generate positive torque around SFB Yaxis. For example, the thruster could be mounted on the negative $\mathrm{Z}$-axis with thrust vector pointing parallel to the negative $\mathrm{X}$-axis. There is also an 'impulsive shot' assumption for simplicity that the thruster firing duration $t_{o n}$ is much smaller than the spin period. The Half-Cone method takes the following sequence of events (also illustrated in Fig.2) where $Z_{0}$ and $Z_{t}$ are the initial and target spinaxes:

(1) For $t<t_{1}$, the spacecraft is in a pure spinning around its $Z_{0}$-axis with no nutation.

(2) At $t=t_{1}$ the spacecraft $X$-axis is perpendicular to the $Z_{0}-Z_{t}$ plane and the thruster generates an angular impulse in positive $\mathrm{Y}$ direction pointing to $Z_{t}$ axis which drives the angular momentum vector away from $Z_{0}$-axis to half the $Z_{0}-Z_{t}$ angle.

(3) For $t>t_{1}$, the spacecraft spin axis starts a precession motion around the intermediate $\boldsymbol{H}$ vector with no torques applied.

(4) When the spin axis reaches the target spin axis $Z_{t}$, a second impulse torque is given to realign the $\boldsymbol{H}$-vector with $Z_{t}$. 
(5) Then the precession is stopped and the Half-Cone manoeuvre is accomplished.

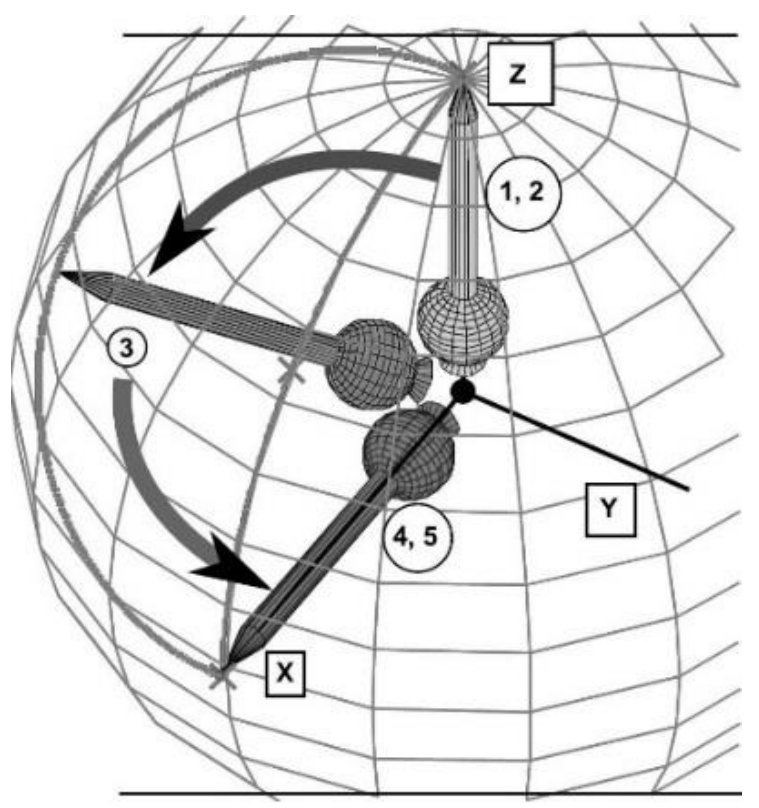

Fig.2. the sequence of events for the Half-Cone Slew control manoeuvre [3]

The constraint experienced by this control algorithm will be with respect to the position of the thruster for the actuation of the second impulse that is also known as the cancellation impulse. The cancellation pulse needed to be in the same magnitude but opposite in direction to the first pulse that is also known as the initiation pulse. In order to provide the needed torque, the thruster will have to rotate $(2 \mathrm{k}+1)$ half revolutions where $\mathrm{k}$ is a non-negative integer representing the total number of revolution(s) the spacecraft will make. As a result, the equation has been derived to accommodate the constraint [3].

$$
t=\frac{\pi}{\omega_{H}}=(2 k+1) \frac{\pi}{\omega_{N}} \Leftrightarrow \omega_{N}=(2 k+1) \omega_{H}
$$

With $\omega_{H}$ and $\omega_{N}$ replaced by Eq. (2) and Eq. (3), the following Eq. (5) is then derived.

$$
\begin{gathered}
\omega_{Z}\left(1-\frac{I_{Z}}{I_{t}}\right)=(2 k+1) \frac{\|\boldsymbol{H}\|}{I_{t}} \Leftrightarrow \\
\omega_{Z}\left(I_{t}-I_{Z}\right)=(2 k+1)\|\boldsymbol{H}\|
\end{gathered}
$$

By referring to the Z- $H$ plane illustrated, the following equation is then derived to show the relationship between $\mathrm{k}$ and the nutation angle, $\theta$.

$$
\begin{array}{r}
\cos (\theta)=\frac{I_{Z} \omega_{Z}}{\|\boldsymbol{H}\|} \Rightarrow \omega_{Z}\left(I_{t}-I_{Z}\right)=(2 k+1) \frac{I_{Z} \omega_{Z}}{\cos (\theta)} \\
\Rightarrow \cos (\theta)=(2 k+1) \frac{I_{Z}}{I_{t}-I_{Z}} \Rightarrow \cos (\theta)=(2 k+1) \frac{\lambda}{1-\lambda}
\end{array}
$$

This relation has shown that with increasing value of $\lambda$, the number of attainable target slew, $\beta$ angle decrease dramatically as $\beta \approx 2 \theta$. However, due to the range of cosine function values, the inertia ratio $\lambda$ is constrained as $0<\lambda<0.5$. The following Fig. 3 explains the constraint further.

For each initiation and the cancellation pulse, the following is used to calculate the angular impulse, $\delta H$.

$$
\delta H=\tan (\theta) I_{Z} \omega_{Z}
$$




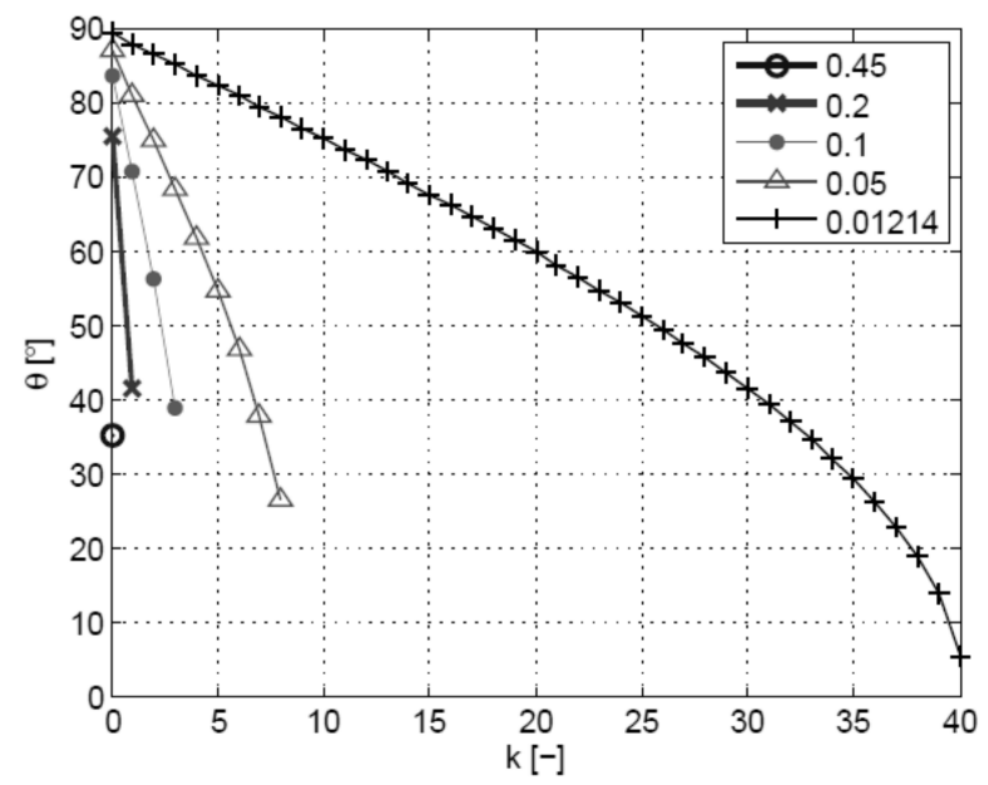

Fig. 3. $\theta$ versus $\mathrm{k}$ for several values of $\lambda$ [3]

\subsection{Sector-Arc Slew(SAS)}

Sector-Arc Slew method is an enhancement of the Half-Cone method. The difference is that it provides additional degree of freedom of the algorithm by not constraining the angular moment to be coplanar with the initial and final spin axis vectors, therefore the nutation angle $\theta$ can be chosen independently from the desired slew angle $\beta$.

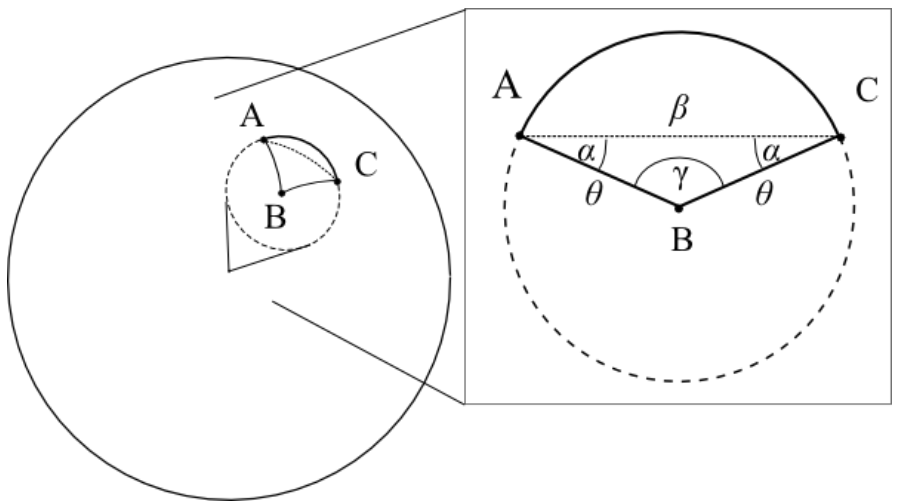

Fig. 4. definition of SAS fundamental angles

SASs can consist of any number of sector arcs. For the purposes of describing the SAS method in detail, we only discuss the slew of only one sector arc. First, we must define the four angles that characterize a single sector arc as described on a unit sphere with origin at point O. As shown in Fig.4, the spin axis unit vector translates from point $\mathrm{A}$ to point $\mathrm{C}$, around the angular momentum vector located at point $\mathrm{B}$. The angles are defined as follows:

- $\quad \theta$ (the angle A-O-B): the nutation angle. This is equal to the arc length from A to $\mathrm{B}$.

- $\quad \beta$ (the angle A-O-C): the slew increment performed by the sector arc. In the case of slew with single sector arc, this represents the target slew angle. This is equal to the arc length from A to C.

- $\quad \alpha$ (the spherical angle B-A-C): the 'azimuth' angle of the sector arc. This defines how far from the idea path the angular momentum vector is positioned during the sector arc.

- $\quad \gamma$ (the spherical angle A-B-C): the angle through which the spin axis precesses about the angular momentum vector during the sector arc. 
Similar with Half-Cone slew, SAS also requires to calculate the thruster time profile to guide the slew. Given the staring slew epoch $t_{0}$, the starting firing time $t_{s}$ and cancelation firing time $t_{f}$ can be calculated by:

$$
\begin{gathered}
t_{s}=t_{0}+t_{A} \\
t_{f}=t_{s}+\Delta t_{A C}
\end{gathered}
$$

The summary of calculations of referred intermediate variables $t_{A}$ and $\Delta t_{A C}$ and four fundamental angles $(\theta, \beta, \alpha, \gamma)$ are provided in Fig. 5. That is all that is needed to implement the algorithm for a general single sector arc SAS slew.

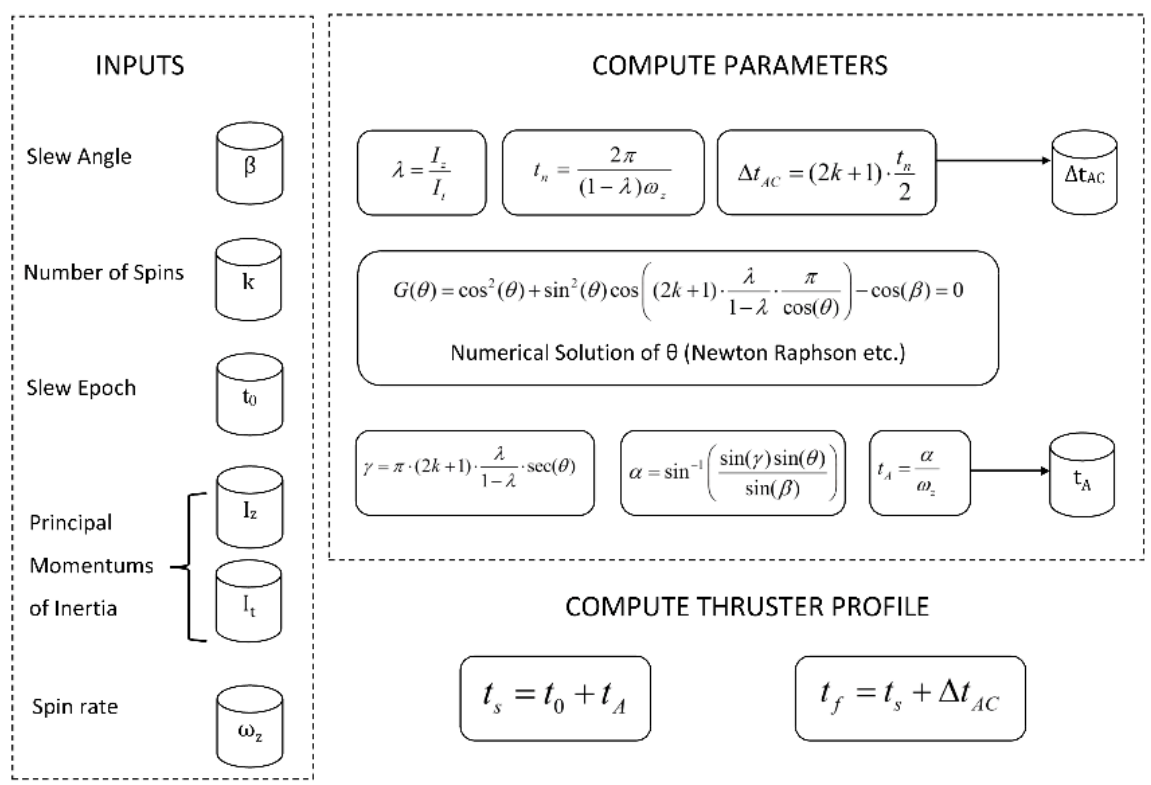

Fig. 5. summary of SAS algorithm

From the $G(\theta)$ equation, $\theta$ cannot be easily solved analytically; however, it is possible to perform a numeric solution of the variable $\theta$ (e.g. via the Newton Raphson method). This step could be implemented on-board via a look-up table if deemed necessary to avoid on-board numerical iteration.

Given a target slew angle $\beta$, the SAS slew can be tuned by choosing the number of spins, $k$, which makes the SAS algorithm optimizable for minimum slew durations, minimum angular impulse magnitude required or least net propellant mass consumed. The parameter $\mathrm{k}$ can be tuned either in advance of the slew based on a priori knowledge of slew conditions or in real-time via on-board solution of the cost-function minimization problem.

\subsection{Slew Performance Measurements}

Slew accuracy is measured by the angular difference between the target attitude and the final attitude. However, the angular momentum is not always aligned with the spin axis attitude after the slew, so there is final precession around the final angular momentum for most cases. This is why the slew error is divided into two parts: angular momentum error $\Delta H$, the angle between target angular momentum $\boldsymbol{H}_{t}$ and final angular momentum $\boldsymbol{H}_{f}$, and the residual nutation angle $\Delta Z$, which is the angle between the final spin axis $\boldsymbol{Z}_{f}$ and $\boldsymbol{H}_{f}$. See Fig. 6. After the completion of the slew, these two errors are independent on the time. 


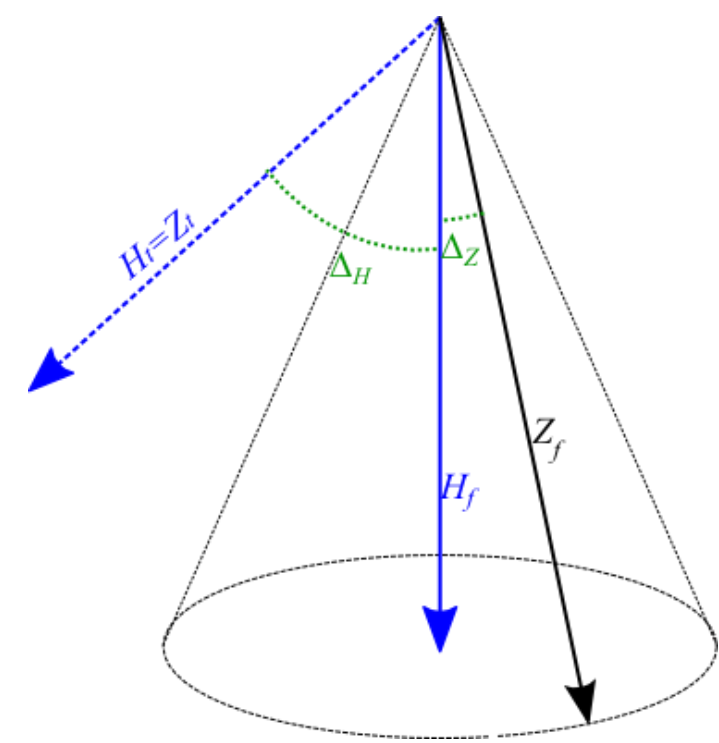

Fig. 6. accuracy: angular momentum error $\Delta H$ and residual nutation angle $\Delta Z$

Since the spin-axis may perform precession after the slew, it is also of great significance that we calculate the maximum and minimum spin-axis error to the target spin-axis attitude $\boldsymbol{Z}_{t}$.

$$
\begin{gathered}
\varepsilon_{\max }=\Delta H+\Delta Z \\
\varepsilon_{\min }=\operatorname{abs}(\Delta H-\Delta Z)
\end{gathered}
$$

\section{Proposed Feedback Half-Cone Slew and Sector-Arc Slew}

In practical space applications, spin-rate perturbation exists all the time; however, the open-loop HalfCone slew and Sector-Arc Slew show great sensitivity to the spin-rate perturbation. Due to lack of attitude and spin-rate feedback, the spin-rate perturbation always leads to great deviation of the initial impulse's direction, which results in large slew error or even the failure of the slew. In order to improve the robustness of Half-Cone slew and Sector-Arc slew method, novel feedback Half-Cone slew algorithm and Sector-Arc Slew algorithm with attitude and spin-rate feedback are proposed in this paper. The philosophy of feedback Half-Cone slew and feedback Sector-Arc slew are to use the mentioned feedback to adjust the timing and duration of the cancelation impulse, aiming at the smallest residual nutation error and angular momentum error. Two techniques are developed: (1) Estimation of the angular momentum $\mathrm{H}$ during the precession; (2) Prediction of the spin-axis attitude of next two rotations of spacecraft.

With the help of the angular momentum estimation and spin-axis attitude prediction, the cancellation impulse's timing and duration can be calculated. The methods for $H$ estimation and attitude prediction are explained in the following sub-sessions. The flow-chart of Feedback HC slew can be seen in Fig.7. Feedback Sector-Arc slew follows the similar flow-chart of feedback Half-Cone, though the initial impulse is calculated as illustrated in Section 3.

To achieve this goal, attitude feedback is taken by Sun sensor and horizon sensors calculated by Earthwidth/Sun angle method. The spin-rate feedback is taken from onboard gyros. 


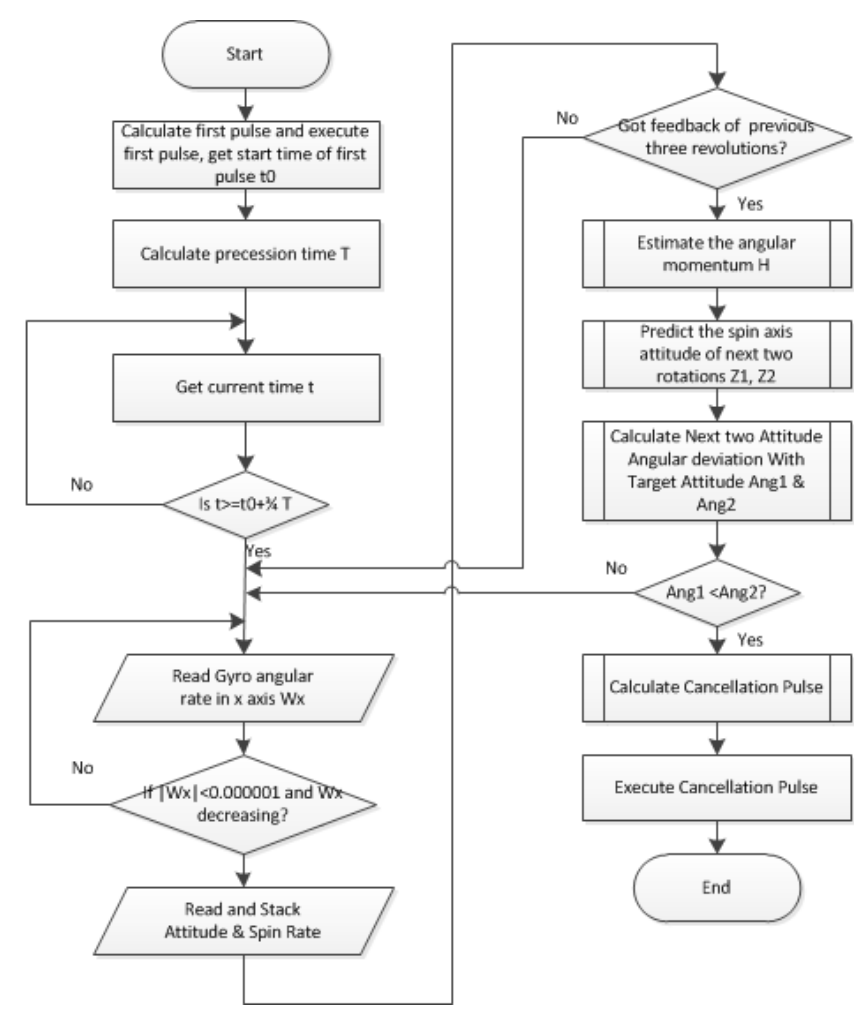

Fig. 7. flow-chart of Feedback Half-Cone Slew algorithm

\subsection{Angular Momentum Estimation}

After the spacecraft finishes at least 3 rotations of spinning during the precession, the angular momentum $\mathrm{H}$ can be estimated. Take $\mathrm{n}$ as the numbers of rotation. Since $\boldsymbol{H}_{n}$ has the same angular deviations with $\boldsymbol{Z}_{n-2}, \boldsymbol{Z}_{n-1}$ and $\boldsymbol{Z}_{n}$, as shown in Fig.8, following equations exist:

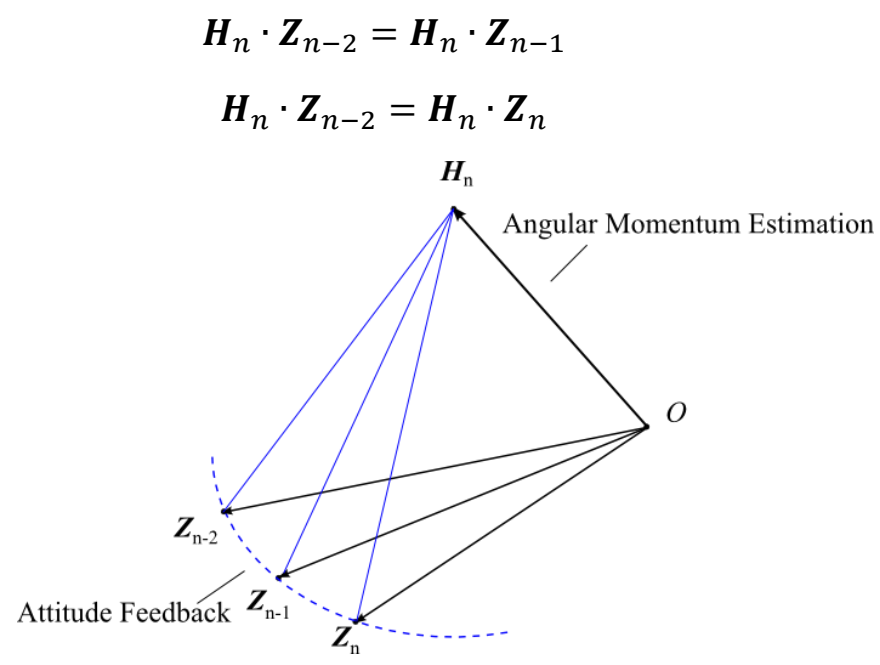

Fig. 8. angular momentum estimation

$\boldsymbol{H}_{n}$ is defined as a unit vector for the convenience of research, which gives:

$$
\left\|\boldsymbol{H}_{n}\right\|=\sqrt{\left(H_{n x}^{2}+H_{n y}^{2}+H_{n z}^{2}\right)}=1
$$

$\boldsymbol{H}_{n}$ is a three-element column vector with three variables. These three variables of the vector can be solved by Eqs. (12) - (14) as a ternary linear equation set. 
Due to the quadratic characteristic of Eqs (14), there will be two solutions for $\boldsymbol{H}_{n}$. These two solutions take mirror-like positions to the spin-axis attitude feedbacks. With the limitation of the Half-cone method, the nutation angle is less than $90^{\circ}$, and this prior knowledge $\boldsymbol{H}_{n} \cdot \boldsymbol{Z}_{n}>1$ is used to eliminate the false solution.

\subsection{Spin-axis Attitude Prediction}

The purpose of attitude prediction is to predict the next two spin-axis attitude after No. $\mathrm{n}$ spin-axis attitude measurement. The normal vector of $Z_{n-1} O H_{n}$ plane is defined as $\boldsymbol{N}_{n-1}$, and it is calculated by:

$$
\boldsymbol{N}_{n-1}=\boldsymbol{Z}_{n-1} \times \boldsymbol{H}_{n}
$$

Similarly, the normal vector $\boldsymbol{N}_{n}$ of $Z_{n} O H_{n}$ plane is calculated by:

$$
\boldsymbol{N}_{n}=\boldsymbol{Z}_{n} \times \boldsymbol{H}_{n}
$$

Therefore, the estimated precession rate at the No. $n$ attitude measurement is:

$$
\left(\omega_{H}\right)_{n}=\operatorname{ang}\left(\boldsymbol{N}_{n}, \boldsymbol{N}_{n-1}\right) / \Delta t_{n-1}
$$

$\Delta t_{n-1}$ is the time interval between No. $\mathrm{n}$ and No.(n-1) attitude measurement. The nutation angle $\theta_{n}$ is calculated by:

$$
\theta_{n}=\operatorname{ang}\left(\boldsymbol{H}_{n}, \boldsymbol{Z}_{n}\right)
$$

Then the estimated spin rate and the body nutation rate at that time are:

$$
\begin{gathered}
\left(\omega_{Z}\right)_{n}=\left(\omega_{H}\right)_{n} \cdot \cos \left(\theta_{n}\right) / \lambda \\
\left(\omega_{N}\right)_{n}=(1-\lambda) \cdot\left(\omega_{Z}\right)_{n}
\end{gathered}
$$

The estimated spin-axis attitude measurement will take place after:

$$
\Delta t_{n}^{*}=\pi /\left(\omega_{N}\right)_{n}
$$

The next two attitude predictions are generated by rotation angles of $\gamma_{n}$ and $2 \gamma_{n}$ around $\boldsymbol{H}_{n}$ from $\boldsymbol{Z}_{n}$, where:

$$
\begin{gathered}
\gamma_{n}=\left(\omega_{H}\right)_{n} \cdot \Delta t_{n}^{*} \\
Q_{n}=\left[\boldsymbol{H}_{n} \cdot \sin \left(\gamma_{n} / 2\right), \cos \left(\gamma_{n} / 2\right)\right]^{\prime} \\
Q_{n+1}=\left[\boldsymbol{H}_{n} \cdot \sin \left(\gamma_{n}\right), \cos \left(\gamma_{n}\right]^{\prime}\right.
\end{gathered}
$$

Therefore, the predicted spin-axis attitudes of next two measurements are:

$$
\begin{gathered}
\boldsymbol{Z}_{n+1}^{*}=\operatorname{DCM}\left(Q_{n}\right) * \boldsymbol{Z}_{n} \\
\boldsymbol{Z}_{n+2}^{*}=\operatorname{DCM}\left(Q_{n+1}\right) * \boldsymbol{Z}_{n}
\end{gathered}
$$

\subsection{Cancellation Impulse Timing and Duration}

After the No. n's spin axis attitude measurement is taken, if the next attitude prediction satisfies:

$$
\gamma_{n}>1.5 * \operatorname{ang}\left(\boldsymbol{Z}_{t}, \boldsymbol{Z}_{n+1}^{*}\right)
$$

it is assumed that the slew will be finished in the next spin revolution. There are two situations for the slew finale: 
a) $\operatorname{ang}\left(\boldsymbol{Z}_{t}, \boldsymbol{Z}_{n+1}^{*}\right)<\operatorname{ang}\left(\boldsymbol{Z}_{t}, \boldsymbol{Z}_{n+2}^{*}\right)$

In this case, a cancellation impulse is required in the next spin period. The purpose of this cancellation impulse is to minimize the final nutation angle by aligning the angular momentum vector with the spinaxis as well as to finish the slew control. Fig.9 illustrates the final cancellation impulse.

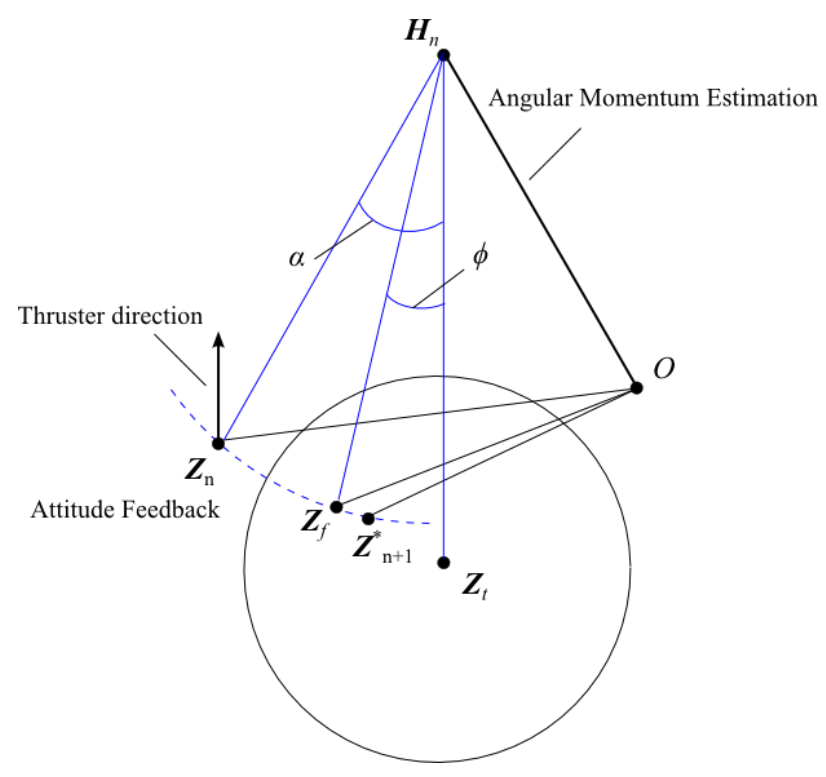

Fig. 9. final cancellation impulse illustration

In order to minimize the final nutation angle, the time interval $\Delta t_{f}$ between the final cancellation impulse and No. $\mathrm{n}$ attitude measurement satisfies:

$$
\begin{gathered}
\phi=\alpha-\left(\omega_{H}\right)_{n} \cdot \Delta t_{f} \\
\phi=2 \pi-\left(\omega_{N}\right)_{n} \cdot \Delta t_{f}
\end{gathered}
$$

Solving $\Delta t_{f}$ from Eqs.(28)-(29) gives:

$$
\Delta t_{f}=(2 \pi-\alpha) /\left(\left(\omega_{N}\right)_{n}-\left(\omega_{H}\right)_{n}\right)
$$

Eq.(30) calculates the time when final cancellation impulses is applied. Due to the feature, Half-Cone slew, the final impulse duration is the same as the initiation impulse.

b) $\operatorname{ang}\left(\boldsymbol{Z}_{t}, \boldsymbol{Z}_{n+1}^{*}\right)>\operatorname{ang}\left(\boldsymbol{Z}_{t}, \boldsymbol{Z}_{n+2}^{*}\right)$

In this case, the slew repeat the attitude measurement in the next spin period and test for the condition as mentioned in Eq. (27).

\section{Simulations and Robustness analysis}

\subsection{Mission scenario}

Simulations were made using MATLAB Simulink. The initial state of the spacecraft is a pure spin around it SFB Z-axis and the SFB frame is coincident with the RI frame at this moment. Table 1 defines the constant used during the simulation. 
Table 1: Input parameters for simulations

\begin{tabular}{|c|c|}
\hline Input parameters & Values \\
\hline Mass M & $15 \mathrm{~kg}$ \\
\hline$I_{x}$ & $5.35416 \mathrm{~kg} \cdot \mathrm{m}^{2}$ \\
\hline$I_{y}$ & $5.35416 \mathrm{~kg} \cdot \mathrm{m}^{2}$ \\
\hline$I_{z}$ & $0.130 \mathrm{~kg} \cdot \mathrm{m}^{2}$ \\
\hline Attitude thruster position & {$\left[\begin{array}{lll}0 & 0 & -1\end{array}\right] \mathrm{m}$} \\
\hline Attitude thruster thrust direction & {$\left[\begin{array}{lll}-1 & 0 & 0\end{array}\right]$} \\
\hline Attitude thruster max thrust & $0.1 \mathrm{~N}$ \\
\hline Spin rate (around $\mathrm{Z}$-axis) & $0.2 \mathrm{rad} / \mathrm{s}$ \\
\hline Initial Euler angles & {$\left[\begin{array}{lll}0 & 0 & 0\end{array}\right]$} \\
\hline Target slew angle & $90^{\circ}$ \\
\hline
\end{tabular}

\subsection{Open-loop Half-Cone Slew and Feedback Half-Cone Slew}

Simulations are done using the same input parameters in Table 1 for both Open-loop Half-Cone Slew and Feedback Half-Cone Slew when $-1 \% \sim 1 \%$ spin-rate perturbations are applied to the slews.

\subsubsection{Slew Performance over spin-rate perturbations and range of target slew angles}

Simulations are done to reveal the robustness of Open-loop Half-cone and Feedback Half-cone slew over-1\% $\sim 1 \%$ spin-rate perturbations and a range of target slew angles. The simulation result can be seen in Fig.10- Fig. 13.
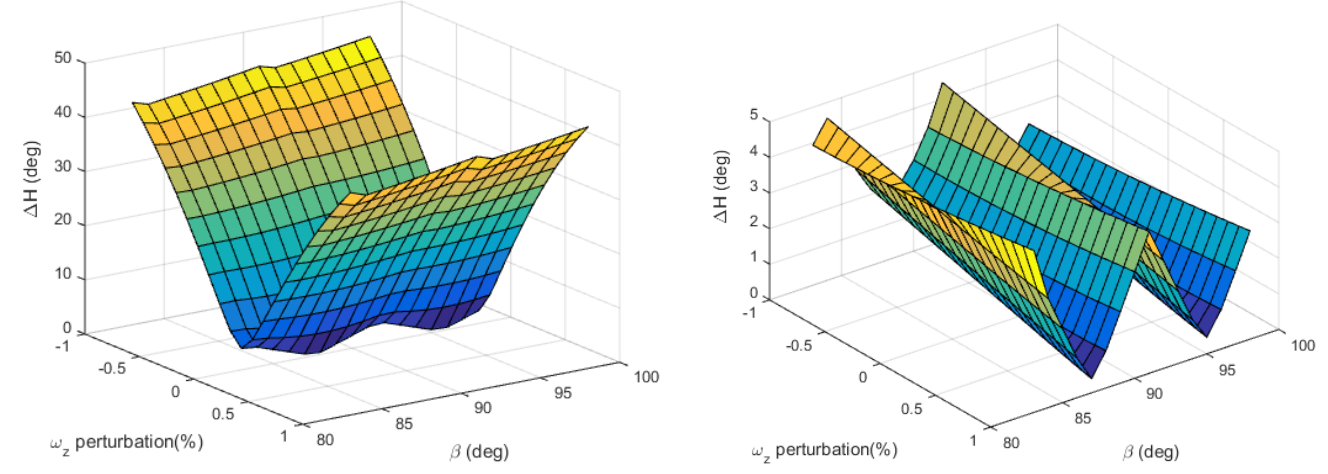

Fig. 10. $\Delta H$ over $\Delta \omega$ and $\beta$ for $\mathrm{OHC}$ and FHC 

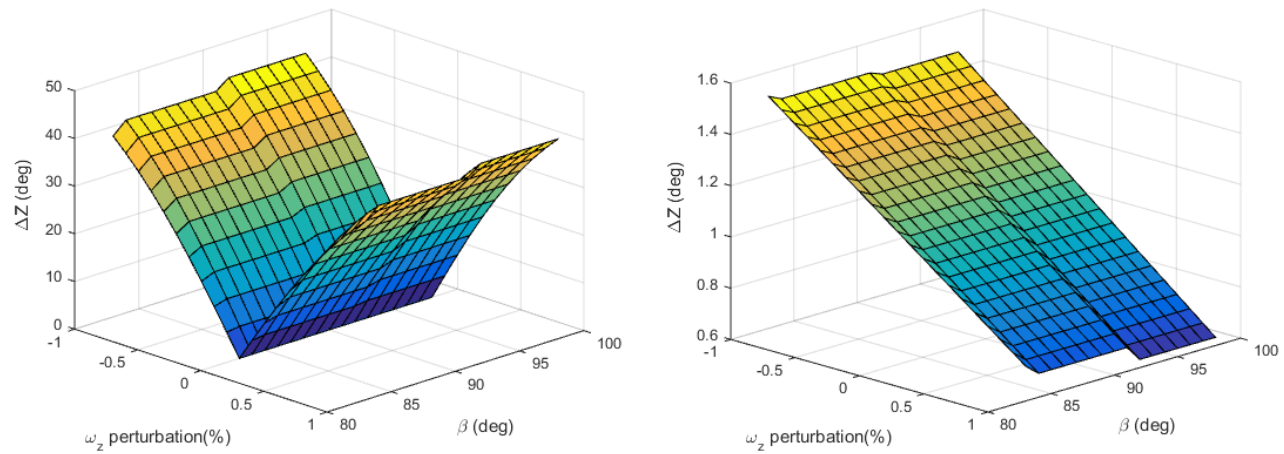

Fig. 11. $\Delta Z$ over $\Delta \omega$ and $\beta$ for $\mathrm{OHC}$ and FHC

It can be seen that even with -1\% 1\% spin rate perturbations, open-loop Half-Cone Slew results in over $40^{\circ}$ of both angular momentum error $\Delta H$ and residual nutation error $\Delta Z$ for different slew angles. For space missions, this scale of slew error is unacceptable and indicates the failure of slew. This is because open-loop Half-cone slew cannot restrain the influence of spin-rate error over the thruster firing timing and this error accumulates over spin revolutions. That is the reason why open-loop Half-cone slew is very sensitive to spin-rate perturbations.

With the help of attitude feedback, feedback Half-cone slew can reduce $\Delta H$ to less than $5^{\circ}$ and $\Delta Z$ to less than $2^{\circ}$ when $-1 \% \sim 1 \%$ spin rate perturbations are applied. It proves that Feedback Half-Cone Slew greatly improves the robustness of half-cone slew and provides reliable slew performance.

It is interesting to see that Feedback Half-Cone Slew gives better slew performance for $\Delta Z$ over positive spin rate perturbations. That is because with greater spin rate, more frequently the attitude feedback is taken and that improves the slew performance.
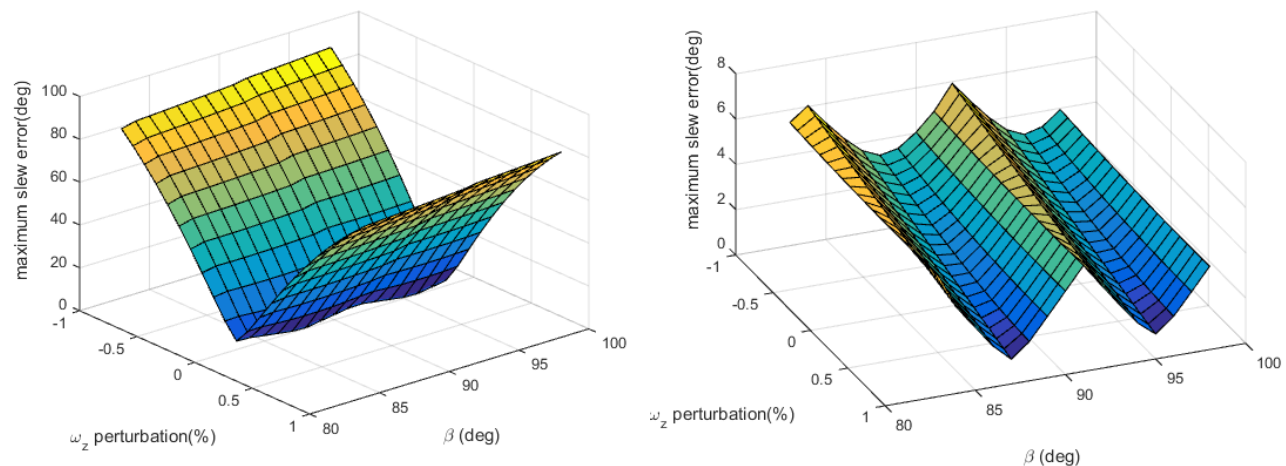

Fig. 12. $\varepsilon_{\max }$ over $\Delta \omega$ and $\beta$ for OHC and FHC
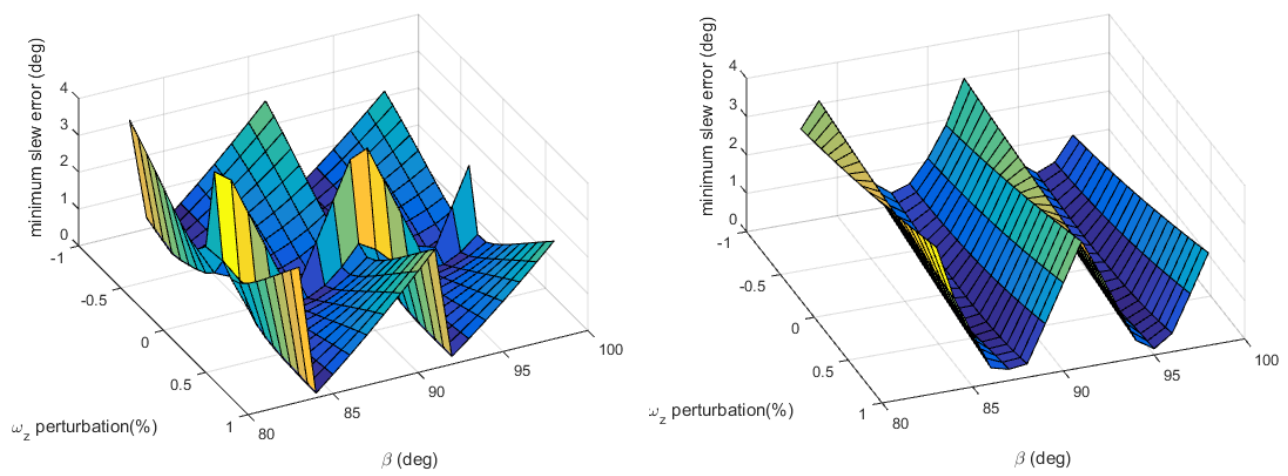

Fig. 13. $\varepsilon_{\min }$ over $\Delta \omega$ and $\beta$ for OHC and FHC 
The overall slew error can be seen in Fig.12. and Fig. 13. Open-loop Half-Cone may have huge $\varepsilon_{\max }$ over $80^{\circ}$. In the meantime, Feedback Half-Cone controls the maximum slew error down to $6^{\circ}$.

\subsubsection{Slew Performance over spin-rate perturbations and range inertia ratios}

More simulations are done to reveal the robustness of Open-loop Half-cone and Feedback Half-cone slew over $-1 \% \sim 1 \%$ spin-rate perturbations and a range of inertia ratios $\lambda$. The simulation result can be seen in Fig.14. Fig. 17.
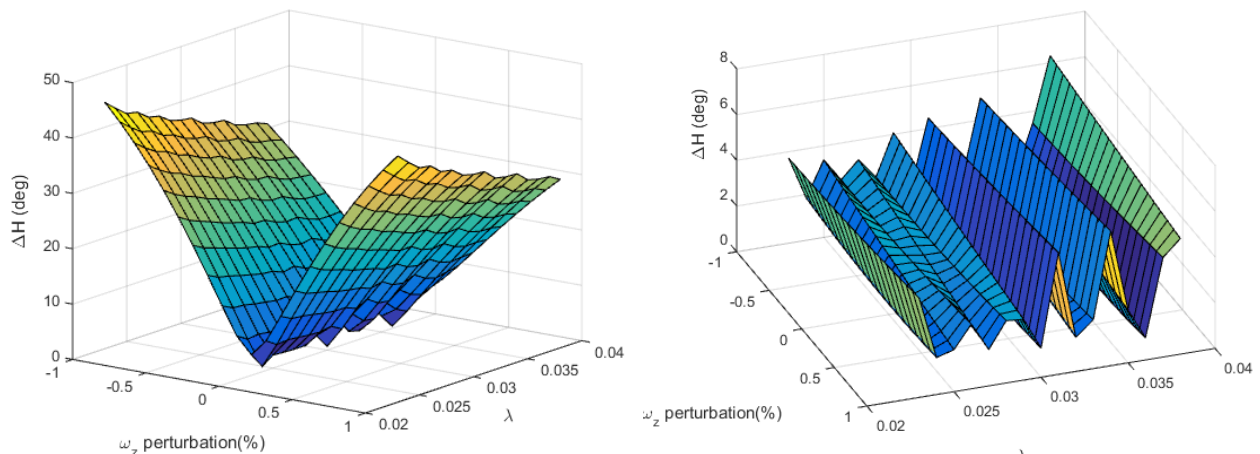

Fig. 14. $\Delta H$ over $\Delta \omega$ and $\lambda$ for $\mathrm{OHC}$ and FHC
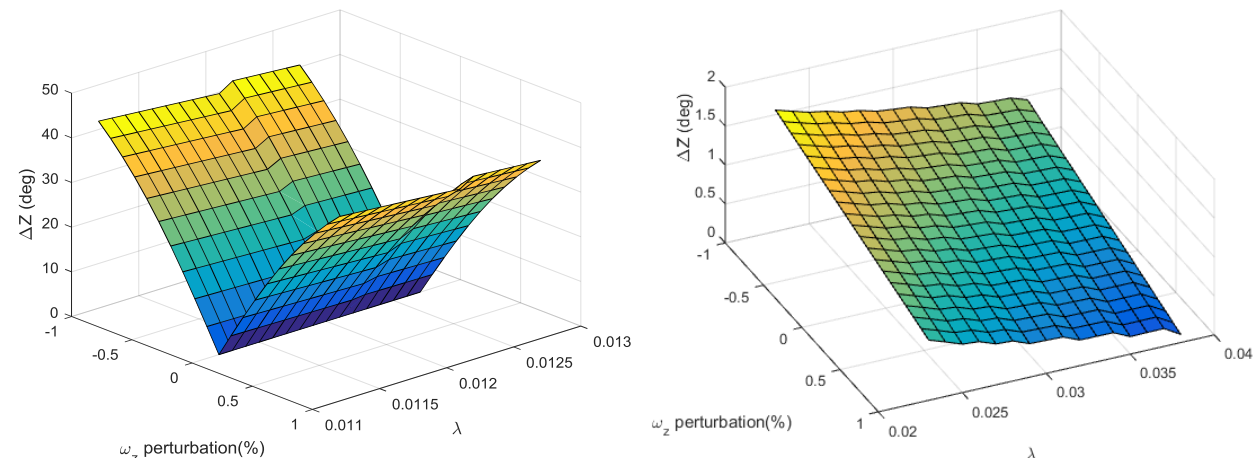

Fig. 15. $1 \Delta Z$ over $\Delta \omega$ and $\lambda$ for $\mathrm{OHC}$ and FHC
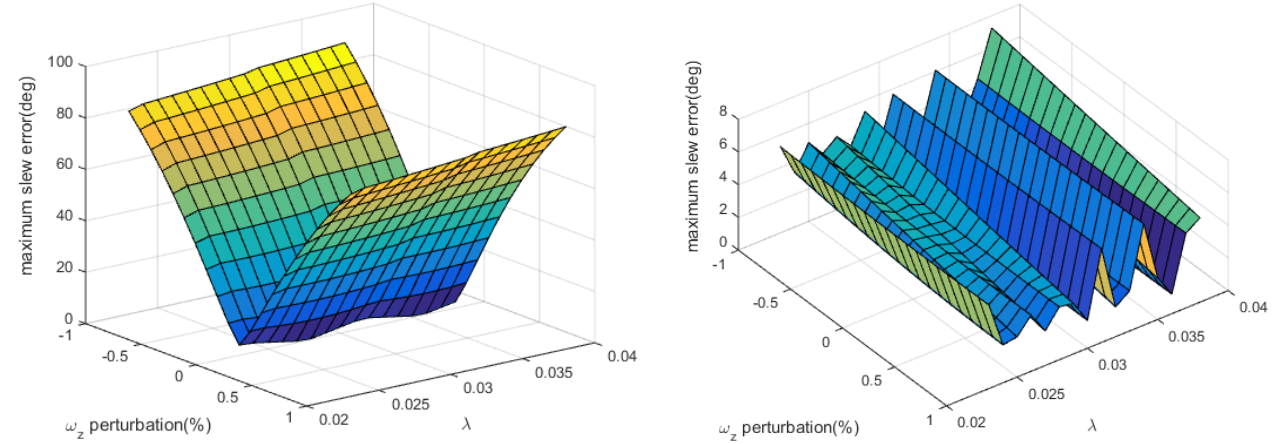

Fig. 16. $\varepsilon_{\max }$ over $\Delta \omega$ and $\lambda$ for $\mathrm{OHC}$ and FHC 

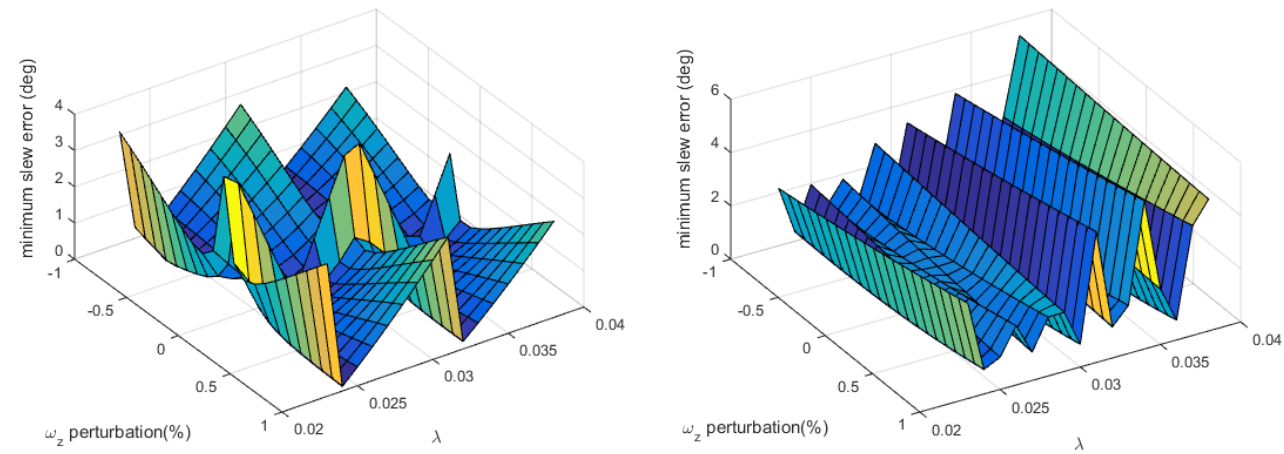

Fig. 17. $\varepsilon_{\min }$ over $\Delta \omega$ and $\lambda$ for $\mathrm{OHC}$ and FHC

Similar results can be seen that Feedback Half-Cone Slew greatly improves the slew robustness over spin-rate perturbations and a range of $\boldsymbol{\lambda}$. Open-loop Half-Cone has huge $\boldsymbol{\varepsilon}_{\max }$ over $80^{\circ}$. In the meantime, feedback Half-Cone controls the maximum slew error down to $7^{\circ}$.

Ups and downs can be seen in this set of simulations as well. That is due to the discontinuity of halfcone method itself, which is described in Section 3.

\subsection{Feedback Sector-arc slew}

\subsubsection{Slew Performance over spin-rate perturbations and range of target slew angles}

Simulations are done to reveal the robustness of feedback Sector-arc slew over-1\% 1\% spin-rate perturbations and a range of target slew angles. The simulation result can be seen in Fig.18. Fig. 21.
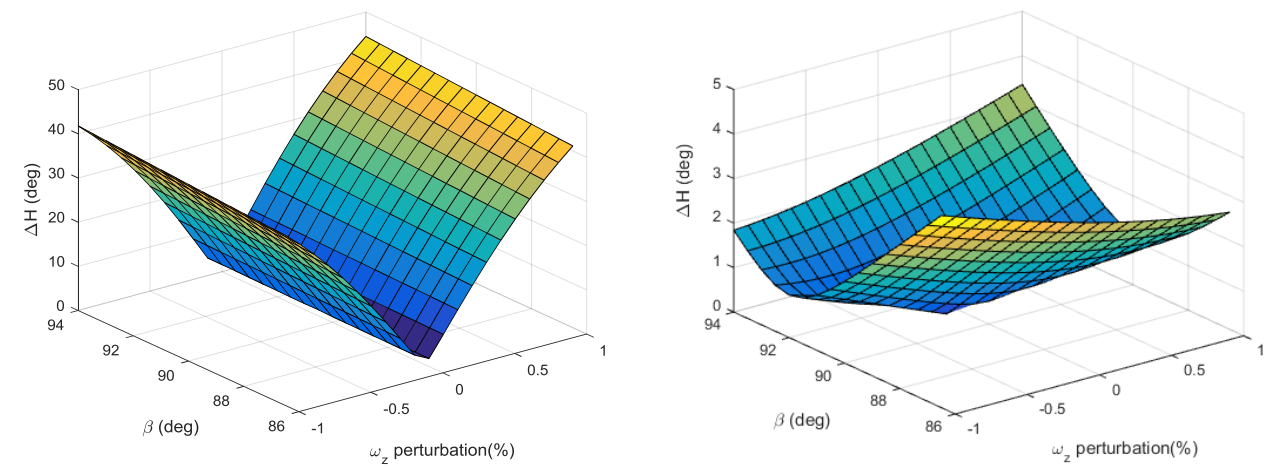

Fig. 18. $\Delta H$ over $\Delta \omega$ and $\beta$ for OSAS and FSAS
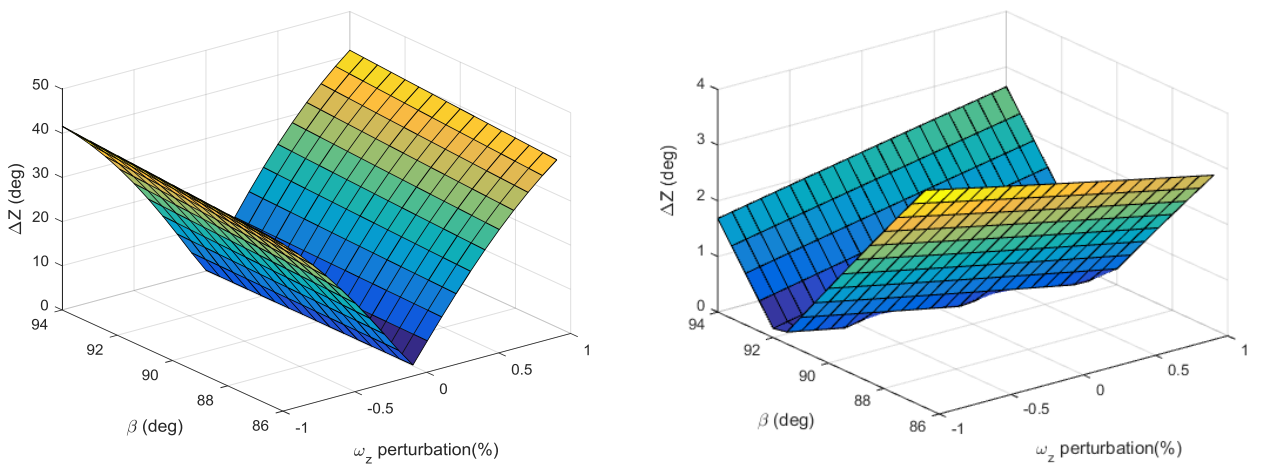

Fig. 2. $\Delta Z$ over $\Delta \omega$ and $\beta$ for OSAS and FSAS 
For $-1 \% \sim 1 \%$ spin rate perturbation, open-loop Sector-Arc Slew results in over $40^{\circ}$ of slew error in both $\Delta H$ and $\Delta Z$ for different slew angles. By introducing attitude feedback, Feedback Sector-Arc Slew reduced the $\Delta H$ down to $4^{\circ}$ and $\Delta Z$ down to $3^{\circ}$.
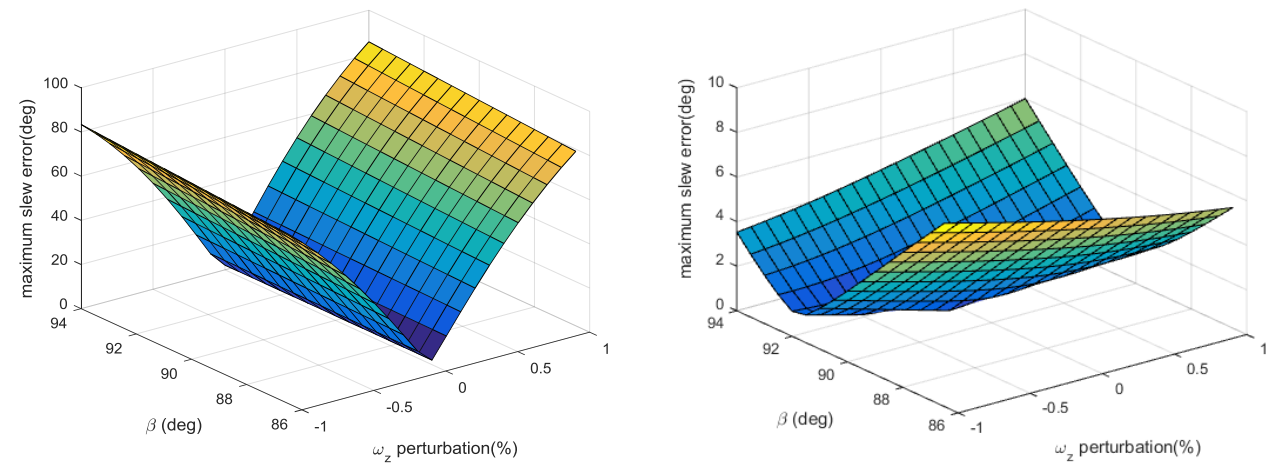

Fig. 20. $\varepsilon_{\text {max }}$ over $\Delta \omega$ and $\beta$ for OSAS and FSAS
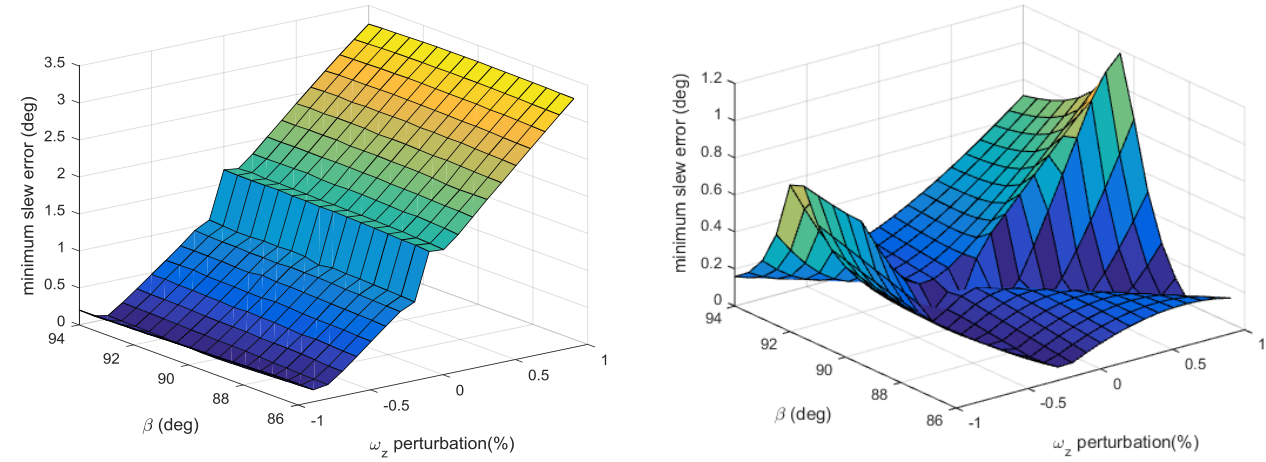

Fig. 21. $\varepsilon_{\min }$ over $\Delta \omega$ and $\beta$ for OSAS and FSAS

The overall slew error can be seen in Fig. 20. and Fig.21. The maximum slew error for open-loop SAS control can reach over $80^{\circ}$ for $1 \%$ spin rate perturbation. In the meantime, the maximum slew error for Feedback SAS is only $6^{\circ}$ when $1 \%$ spin rate perturbation.

\subsubsection{Slew Performance over spin-rate perturbations and range inertia ratios}

More simulations are done for open-loop SAS and Feedback SAS over-1\% 1\% spin-rate perturbations and a range of $\lambda$. The simulation result can be seen in Fig.22. $\sim$ Fig. 25.
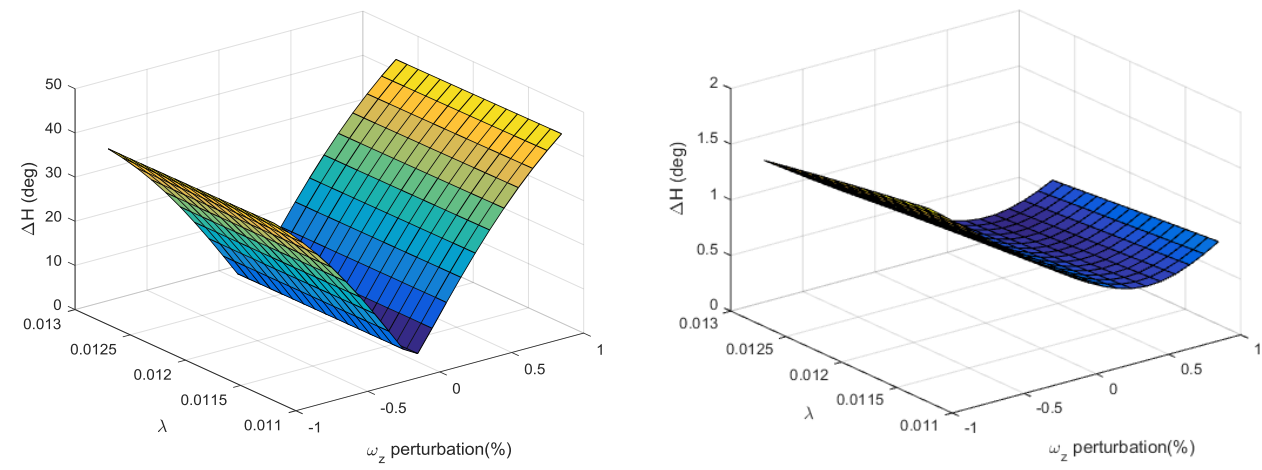

Fig. 22. $\Delta H$ over $\Delta \omega$ and $\lambda$ for OSAS and FSAS 

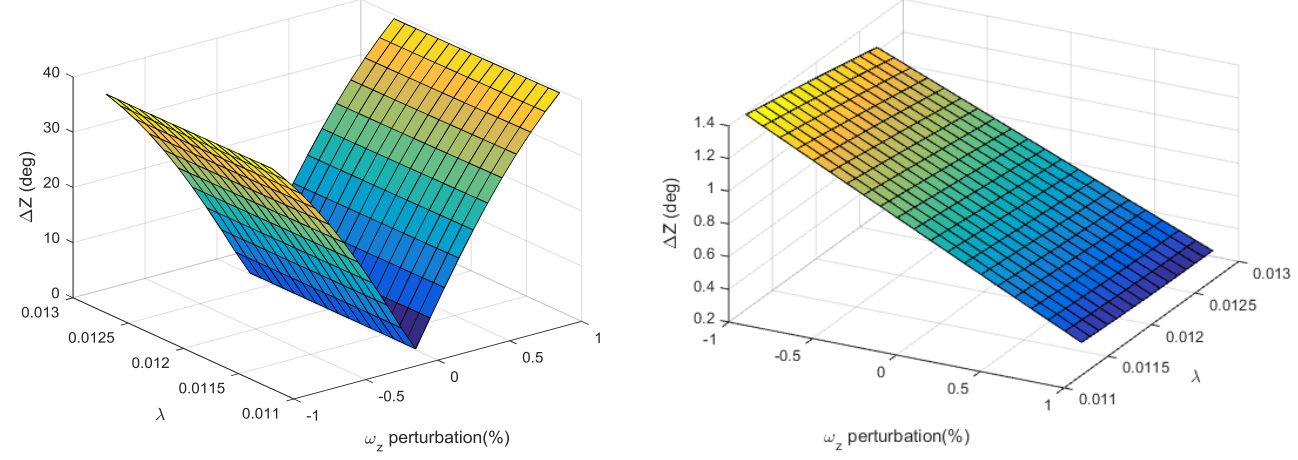

Fig. 23. $\Delta Z$ over $\Delta \omega$ and $\lambda$ for OSAS and FSAS
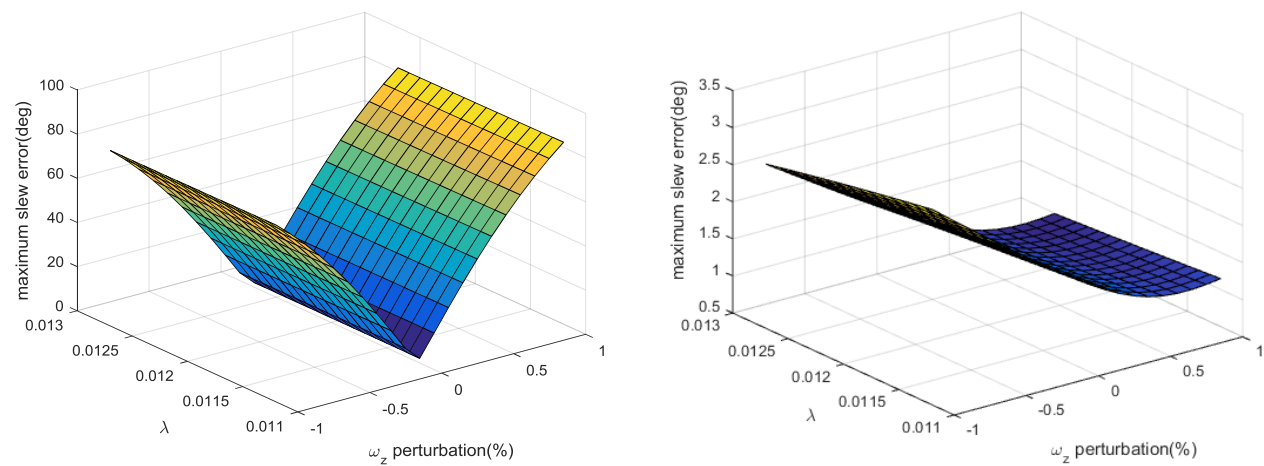

Fig. 24. $\varepsilon_{\max }$ over $\Delta \omega$ and $\lambda$ for OSAS and FSAS
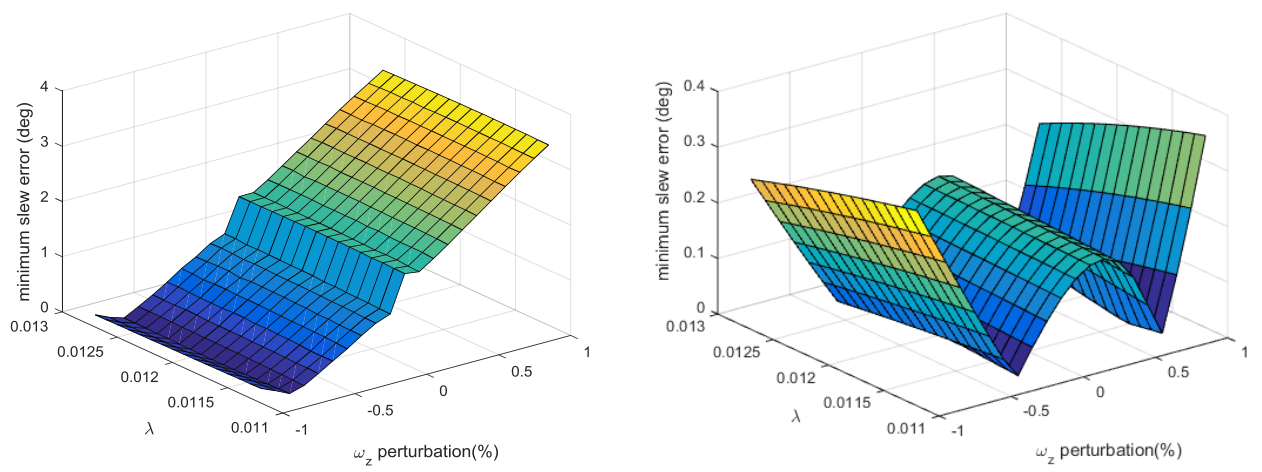

Fig. 25. $\varepsilon_{\min }$ over $\Delta \omega$ and $\lambda$ for OSAS and FSAS

For this set of simulations, reliable slew performance is witnessed with $\Delta H$ reduced down to $1.3^{\circ}, \Delta Z$ down to $1.4^{\circ}, \varepsilon_{\max }$ down to $2.5^{\circ}$ and $\varepsilon_{\min }$ down to $0.3^{\circ}$.

\subsection{Comparison between Feedback Half-Cone Slew and Feedback Sector-Arc Slew for large perturbations}

Feedback Half-cone slew and Sector-arc slew have greatly improved the slew robustness over spin-rate perturbations of $-1 \% \sim 1 \%$. It is also of great interest to see how they perform over larger spin perturbations. Simulations are done for both slew algorithms with the spin-rate perturbation of $15 \% \sim 15 \%$, which is an extremely cruel situation for space missions. The results are shown in Fig. 26.

With this cruel perturbation, Feedback Half-Cone shows even better slew performance than Sector-Arc Slew when the perturbation is over $2 \%$. That is because greater spin-rate perturbation has more influence over the numerical solutions of Sector-Arc Slew. Due to the complex numerical method (e.g. 
via the Newton Raphson method) used in Sector-Arc Slew, FSAS is more sensitive to large spin-rate error.
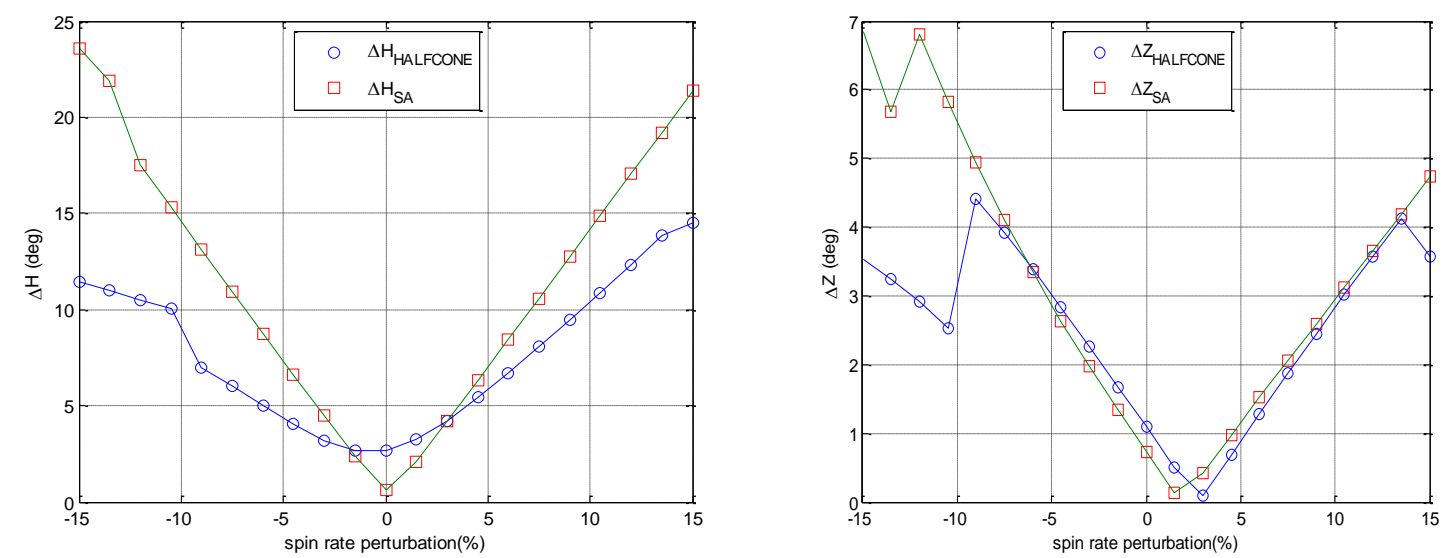

Fig. 26. Slew Performance over -15\% 15\% spin-rate perturbations for FHC and FSAS

6. Conclusion

This paper has introduced novel Feedback Half-Cone slew and Sector-Arc Slew algorithms for prolate spinners' attitude manoeuvre using single-thruster. The algorithms build on open-loop Half-Cone and Sector-Arc Slew methods' philosophy and use attitude feedback and angular velocity information to improve the slew robustness over spin-rate perturbations. The initial impulses of these newly developed algorithms follow the similar calculation as corresponding open-loop methods. Then attitude feedback contributes to angular momentum estimation and spin-axis attitude prediction after that. At the end of slew, a new cancellation impulse design results in great improvement of slew performance based on angular momentum estimation and spin-axis attitude prediction. These two novel slew algorithms are verified by MATLAB Simulink simulations. The simulation results show that Feedback Half-Cone Slew and Feedback Sector-Arc slew greatly improve the slew robustness over spin-rate perturbations. With the comparisons between these two novel slew algorithms, feedback Sector-arc slew shows better robustness over spin-rate perturbations within $-2 \%$ 2\%, while Feedback Half-Cone slew has better performance with spin rate perturbations over $2 \%$ due to the great influence of that over numerical method used by Feedback Sector-Arc Slew. In conclusion, the novel Feedback Half-Cone Slew and Feedback Sector-Arc slew algorithms greatly improve the slew performance over spin-rate perturbations. Future work can include further robustness analysis of the slew algorithms, and the hardware verification of these algorithms.

\section{References}

[1] H. Shiraishi, S. Tanaka, A. Fujimura, H. Hayakawa, The Present Status of the Japanese Penetrator Mission: Lunar-A, Advances in Space Research, 42(2008)386-393.

doi: 10.1016/j.asr.2007.08.022 
[2] Y. Gao, A. Phipps, M. Taylor, I.A. Crawford, A.J. Ball, L. Wilson, D. Parker, M. Sweeting, A.D.S. Curiel, P. Davies, A. Baker, W.T. Pike, A. Smith, and R. Gowen, Lunar science with affordable small spacecraft technologies: MoonLITE and Moonraker, Planetary and Space Science, 56(2008)368-377.

doi: 10.1016/j.pss.2007.11.005

[3] R. Raus, Y. Gao, and M. Watt, Review and Analysis of Single-Thruster Attitude Control Algorithms for Spinning Spacecraft, in International Astronautical Congress 2010 Proceedings. Presented at IAC2010, IAC-10-C1_3_7.

[4] R. Raus, Y. Gao, Y.H. Wu, and M. Watt, Analysis of State-of-the-art Single-Thruster Attitude Control Techniques for Spinning Penetrator, Acta Astronautica, 76(2012)60-78. doi: 10.1016/j.actaastro.2012.02.014

[5] Y.H. Wu, Y. Gao, R. Raus, Tradeoff Analysis of Attitude-Control Slew Algorithms for Prolate Spinner, Journal of Guidance, Control, and Dynamics, 35(2012) 1143-1157. doi: $10.2514 / 1.55619$

[6] Y.H. Wu, Y. Gao, R. Raus and M. Watt, Single thruster attitude control software simulator for spinning spacecraft, IEEE Aerospace Conference 2012, 3-10 March, Big Sky, MT, USA.

[7] J. Si, Y Gao, A. Chanik, Slew Control of Prolate Spinners Using single Magnetorquer, Journal of Guidance, Control, and Dynamics, 39(2015)719-727. doi: 10.2514/1.G001035

[8] S. Kenyon, C.P. Bridges, M. Liddle, R. Dyer, J. Parsons, D. Feltham, R. Taylor, D. Mellor, A. Schofield, and R. Linehan, STRaND-1: Use of a $\$ 500$ Smartphone as the Central Avionics of a Nanosatellite, 62nd International Astronautical Congress 2011, (IAC-11).

[9] R. Raus, Y. Gao, Y.H. Wu, and M. Watt, Robustness Analyses of Attitude Slew Manoeuvre for Spinning Penetrator Spacecraft, 63rd International Astronautical Congress 2012, 01-05 Oct, Naples, Italy.

[10] J.R. Wertz, Spacecraft Attitude \& Determination Control, Kluwer Academics Publisher, 1978.

[11] A.S. Badrakalimuthu, Autonomous attitude determination for spin based spacecrafts using Sun sensors, M. Sc. Thesis, School of Electronics and Physical Sciences, Department of Electronic Engineering, University of Surrey, UK, 2007.

[12] A.M. Rad, J.H. Nobari, A.A. Nikkha, Optimal attitude and position determination by integration of INS, star tracker, and horizon sensor, IEEE Aerospace and Electronic Systems Magazine, 29(2014)20-33. doi: 10.1109/MAES.2014.130093

[13] J. Si, Y Gao, A. Chanik, Slew Performance of Feedback Half-Cone Algorithm for Prolate Spinners Using SingleThruster, $3^{\text {rd }}$ CEAS EuroGNC Conference 2015, 13-15 April, Toulouse, France. 